\title{
Clarifying the solar wind heat flux instabilities
}

\author{
S. M. Shaaban, ${ }^{1,2 \star}$ M. Lazar ${ }^{1,3}$ and S. Poedts ${ }^{1}$ \\ ${ }^{1}$ Centre for Mathematical Plasma Astrophysics, KU Leuven, Celestijnenlaan 200B, B-3001 Leuven, Belgium \\ ${ }^{2}$ Theoretical Physics Research Group, Physics Department, Faculty of Science, Mansoura University, 35516, Mansoura, Egypt \\ ${ }^{3}$ Institut für Theoretische Physik, Lehrstuhl IV: Weltraum- und Astrophysik, Ruhr-Universität Bochum, D-44780 Bochum, Germany
}

Accepted 2018 June 11. Received 2018 June 04; in original form 2018 April 19

\begin{abstract}
In the solar wind, electron velocity distributions reveal two countermoving populations that may induce electromagnetic (EM) beaming instabilities known as heat flux instabilities. Depending on plasma parameters two distinct branches of whistler and firehose instabilities can be excited. These instabilities are invoked in many scenarios, but their interplay is still poorly understood. An exact numerical analysis is performed to resolve the linear Vlasov-Maxwell dispersion and characterize these two instabilities, e.g. growth rates, wave frequencies, and thresholds, enabling to identify their dominance for conditions typically experienced in space plasmas. Of particular interest are the effects of suprathermal Kappa-distributed electrons that are ubiquitous in these environments. The dominance of whistler or firehose instability is highly conditioned by the beam-core relative velocity, core plasma beta, and the abundance of suprathermal electrons. Derived in terms of relative drift velocity the instability thresholds show an inverse correlation with the core plasma beta for the whistler modes, and a direct correlation with the core plasma beta for the firehose instability. Suprathermal electrons reduce the effective (beaming) anisotropy inhibiting the firehose modes while the whistler instability is stimulated.
\end{abstract}

Key words: instabilities - plasmas - methods: numerical-solar wind.

\section{INTRODUCTION}

Beaming electrons, also known as strahl, escape from the electrostatic potential of the Sun and are observed in the solar wind moving along the magnetic field lines (Feldman et al. 1975, 1978; Pilipp et al. 1987; Lin 1998; Pierrard, Maksimovic \& Lemaire 2001). Guided by the magnetic fields these beams may also provide information about the magnetic field topology in interplanetary space (Pagel, Crooker \& Larson 2005). The electron strahl evolves broadening its pitch-angle width (Hammond et al. 1996; Pagel et al. 2007; Anderson et al. 2012) and loosing intensity with heliospheric distance (Maksimovic et al. 2005; Štverák et al. 2009). In the absence of binary collisions, the major role can be played by the waveparticle scattering, acting to broaden the strahl and involving, most probably, the enhanced fluctuations generated by the beam-plasma instabilities (Gary et al. 1975; Gary 1985; Scime et al. 1994; Pistinner \& Eichler 1998; Gary \& Li 2000; Lacombe et al. 2014; Saeed et al. 2017a). Numerical simulations have confirmed the efficiency of magnetosonic-whistler waves (Vocks et al. 2005; Saito \& Gary 2007) and the electrostatic beam-driven fluctuations (Pavan et al. 2013). After 5.5 au the strahl populations is largely scattered (Graham et al. 2017), building up the less anisotropic halo component

^E-mail: shaaban.mohammed@kuleuven.be of electrons (Gurgiolo et al. 2012), although signatures of this component have been detected even at $10 \mathrm{au}$ (Walsh et al. 2013).

Closer to the Sun the strahl carries the electron heat flux and the electromagnetic (EM) instabilities driven by this beaming component are commonly known as heat flux instabilities (Gary et al. 1975; Gary \& Li 2000; Saeed et al. 2017a, b). Measured electron heat fluxes (Scime et al. 1994; Bale et al. 2013) are lower than Spitzer-Härm predictions (Spitzer \& Härm 1953), suggesting the existence of a regulation mechanism for the heat flux by the kinetic beam-plasma instabilities. This paper presents new results attempting to clarify the interplay of heat flux instabilities, which may destabilize two distinct EM modes, namely, the right-handed (RH) polarized whistler (or electron-cyclotron) modes destabilized for relatively low beaming velocities, and the left-handed (LH) polarized electron firehose instability driven by more energetic beams (Gary 1985). Both these instabilities exhibit maximum growth rates in the magnetic field direction (Gary 1993), and are usually studied in the proton rest frame, where the velocity distribution of electrons combines a sunward moving bulk or core component and an antisunward drifting strahl (which fulfill the zero net current condition). Under a broad range of parameters the whistler heat flux (WHF) instability has the lower beam speed threshold, and maximum growth rates higher than the firehose heat flux (FHF) modes (Gary 1985).

Whistler beaming instability is indeed more often invoked as a plausible mechanism of regulation of the electron heat flux in 
space plasmas (Levinson \& Eichler 1992; Gary 1993; Pistinner \& Eichler 1998). This is somehow surprising, given the fact that whistlers are known to be destabilized by an excess of kinetic (free) energy in a direction perpendicular to the magnetic field (e.g. temperature anisotropy $T_{\perp}>T_{\text {II }}$ of electrons), while parallel beams (or relative drifting) of electrons would be more susceptible to the non-resonant firehose-like instabilities. But this may explain a series of recent interpretations that question the existence of whistler modes (Saeed et al. 2017a), and motivated also us to revisit the heat flux instabilities. Our present investigation is intended to describe the interplay of WHF and FHF instabilities, in particular, their regimes of dominance, and the regime of transition where both instabilities may coexist and compete. The nature of instability is dictated by the velocity distributions of plasma particles. Thus, FHF instability is driven by the counter-beaming electrons, but only one of these populations is involved in the resonant excitation of whistlers. Protons are in general non-resonant, and the WHF instability is therefore independent of the electron(-core)-proton temperature ratio $T_{c} / T_{i}$. However, protons may become resonant and whistlers dependent of $T_{c} / T_{i}$ for sufficiently high values of the electron (core) plasma beta, i.e. $\beta_{c} \equiv 8 \pi n k_{B} T_{c} / B_{0}^{2} \gtrsim 5$ (Gary \& Li 2000), a condition only marginally satisfied in the solar wind (Štverák et al. 2008).

On the other hand, standard Maxwellian representation from the early studies (Gary et al. 1975; Gary 1985, 1993) need to be updated in accord with the observations. The electron heat flux is transported away from the solar corona by the suprathermal electrons with energies $E \sim 80 \mathrm{eV}$ (Pagel et al. 2005) and well described by a drifting Kappa (Maksimovic et al. 2005; Nieves-Chinchilla \& Viñas 2008; Štverák et al. 2009). Moreover, in kinetic simulations the heat flux is enhanced in the presence of suprathermal beam as the power index $\kappa$ decreases (Landi \& Pantellini 2001). Despite these expectations, recent studies modelling the electron strahl with a drifting Kappa have found that growth rates and wave frequencies of heat flux instabilities do not vary with the power index $\kappa$ (Saeed et al. 2017a). We have revisited these effects from a different point of view, which enabled a realistic interpretation of suprathermal electrons and their implication. Quantified by the lower values of $\kappa$, the abundance of suprathermals and their effects can be described only by contrasting with Maxwellian limit $(\kappa \rightarrow \infty)$ of lower temperature (Lazar, Poedts \& Fichtner 2015; Lazar, Fichtner \& Yoon 2016). It becomes thus possible to show that kinetic anisotropy instabilities are stimulated by the suprathermals, confirming the additional free energy of these populations (Lazar et al. 2015; Shaaban et al. 2016; Lazar et al. 2017a; Shaaban et al. 2018).

The manuscript is organized as follows: In Section 2 we introduce the velocity distributions for electrons, with two countermoving populations reproducing the core and suprathermal strahl observed in the solar wind. In order to facilitate the analysis and unveil basic properties of heat flux instabilities, both electron populations are considered with isotropic temperatures, and the effects of protons are minimized by assuming them an isotropic neutralizing background. The strahl is generically described by a drifting Kappa, enabling to retract and complete previous results, inclusive for a standard drifting-Maxwellian strahl (in the limit of large power index $\kappa \rightarrow \infty)$. Heat flux instabilities are discussed in detail in Sections 3 and 4, analysing the effects of the beaming velocity, core beta, and the beam suprathermal population on the unstable solutions of whistler and electron firehose modes. In Section 5 we derive the threshold conditions for both instabilities and identify the regimes of their dominance. The results are summarized in Section 4.

\section{THEORY}

In the solar wind, the electron velocity distribution functions (VDFs) reveal the existence of two components, namely, a thermal dense core and a suprathermal component drifting along the magnetic field lines, under the influence of the field-aligned strahl (Pierrard et al. 2001; Maksimovic et al. 2005). Here we adopt the VDF models in Saeed et al. (2017a) (see also references therein) with a dual structure for the electrons combining a core (subscript c) and a beaming (subscript b) component:

$f_{e}\left(v_{\|}, v_{\perp}\right)=\eta f_{c}\left(v_{\|}, v_{\perp}\right)+\delta f_{b}\left(v_{\|}, v_{\perp}\right)$,

where $॥$ and $\perp$ denote (gyrotropic) directions parallel and perpendicular to the magnetic field, $\delta=n_{b} / n_{0}$ and $\eta=1-\delta$ are the beam and core density contrast, respectively, and $n_{0}$ is the total electron number density. In a working frame fixed to protons (solar wind referential), these two populations are countermoving with opposite drifting velocities, and the distribution can be interpreted as a superposition of a drifting-Maxwellian core with a drifting velocity $U_{c}$, plus a drifting-Kappa beam with an opposite drifting velocity $U_{b}$.

Thus, for the core population we assume a drifting-Maxwellian distribution function

$f_{c}\left(v_{\|}, v_{\perp}\right)=\frac{1}{\pi^{3 / 2} \alpha_{\perp}^{2} \alpha_{\|}} \exp \left(-\frac{\left(v_{\|}+U_{c}\right)^{2}}{\alpha_{\|}^{2}}-\frac{v_{\perp}^{2}}{\alpha_{\perp}^{2}}\right)$,

with thermal velocities $\alpha_{11, \perp}$ defined by the temperature components, as the second-order moments of the distribution

$T_{\|}=\frac{m_{e}}{k_{B}} \int d \mathbf{v} v_{\|}^{2} f_{c}\left(v_{\|}, v_{\perp}\right)=\frac{m_{e} \alpha_{\|}^{2}}{2 k_{B}}$

$T_{\perp}=\frac{m_{e}}{2 k_{B}} \int d \mathbf{v} v_{\perp}^{2} f_{c}\left(v_{\|}, v_{\perp}\right)=\frac{m_{e} \alpha_{\perp}^{2}}{2 k_{B}}$.

The beam component is described by a drifting Kappa

$f_{b}\left(v_{\|}, v_{\perp}\right)=\frac{1}{\pi^{3 / 2} \theta_{\perp}^{2} \theta_{\|}} \frac{\Gamma(\kappa+1)}{\Gamma(\kappa-1 / 2)}\left[1+\frac{\left(v_{\|}-U_{b}\right)^{2}}{\kappa \theta_{\|}^{2}}+\frac{v_{\perp}^{2}}{\kappa \theta_{\perp}^{2}}\right]^{-\kappa-1}$

with parameters $\theta_{e, \|, \perp}$ defined by the kinetic temperatures

$T_{\|}^{K}=\frac{2 \kappa}{2 \kappa-3} \frac{m_{e}}{2 k_{B}} \theta_{\|}^{2}=\frac{2 \kappa}{2 \kappa-3} T_{\|}^{M}>T_{\|}^{M}$,

$T_{\perp}^{K}=\frac{2 \kappa}{2 \kappa-3} \frac{m e}{2 k_{B}} \theta_{\perp}^{2}=\frac{2 \kappa}{2 \kappa-3} T_{\perp}^{M}>T_{\perp}^{M}$,

which are assumed $\kappa$-dependent and implicitly higher than their Maxwellian limits (Lazar et al. 2015, 2017b). Protons (subscript p) are heavier and can be assumed Maxwellian and isotropic, enabling to isolate the effects of electrons. The electron-proton plasma is quasi-neutral, $n_{p} \approx n_{e}=n_{c}+n_{b}$, with zero net current $n_{c} U_{c}+n_{b} U_{b}$ $=0$.

We consider the general dispersion relation of the EM modes propagating parallel $\left(\mathrm{k} \times \mathrm{B}_{0}=0\right)$ to the uniform magnetic field $\left(\mathrm{B}_{0}\right)$, e.g. in Lazar et al. (2018),

$$
\begin{aligned}
\frac{k^{2} c^{2}}{\omega^{2}}= & +\frac{4 \pi^{2}}{\omega^{2}} \sum_{a=c, b, p} \frac{e_{a}}{m_{a}} \int_{-\infty}^{\infty} \frac{d v_{\|}}{\omega-k v_{\|} \pm \Omega_{a}} \int_{0}^{\infty} d v_{\perp} v_{\perp}^{2} \\
& \times\left[\left(\omega-k v_{\|}\right) \frac{\partial f_{a}}{\partial v_{\perp}}+k v_{\perp} \frac{\partial f_{a}}{\partial v_{\|}}\right] .
\end{aligned}
$$


For our three-component plasma the equation reduces to

$$
\begin{aligned}
\tilde{k}^{2}= & \frac{\tilde{w}}{\tilde{k} \sqrt{\mu \beta_{p}}} Z\left(\frac{\mu \tilde{w} \pm 1}{\tilde{k} \sqrt{\mu \beta_{p}}}\right)+\eta \frac{\left(\tilde{w}+u_{c} \tilde{k}\right)}{\tilde{k} \sqrt{\beta_{c}}} Z\left(\frac{\tilde{w} \mp 1+u_{c} \tilde{k}}{\tilde{k} \sqrt{\beta_{c}}}\right) \\
& +\delta \frac{\left(\tilde{w}-u_{b} \tilde{k}\right)}{\tilde{k} \sqrt{\beta_{b}}} Z_{\kappa}\left(\frac{\tilde{w} \mp 1-u_{b} \tilde{k}}{\tilde{k} \sqrt{\beta_{b}}}\right),
\end{aligned}
$$

where $\tilde{k}=k c / \omega_{p, e}$ is the normalized wave number, $\tilde{w}=\omega /\left|\Omega_{e}\right|$ is the normalized wave frequency, $\mu$ is the proton-electron mass contrast, $\beta_{c}$ and $\beta_{b}$ are the core and beam plasma beta, respectively, $\delta=n_{b} / n_{0}$ and $\eta=1-\delta$ are the beam and the core density contrast, respectively, $u_{b}=U_{b} \omega_{p, e} /\left(c \Omega_{e}\right)$ and $u_{c}=\delta u_{b} /(1-\delta)$ are the beam and the core relative velocities, \pm denote the right-handed $(\mathrm{RH})$ and left-handed (LH) circular polarizations, respectively, $Z\left(\xi_{a}^{ \pm}\right)$is the plasma dispersion function (Fried \& Conte 1961)

$$
Z\left(\xi_{a}^{ \pm}\right)=\frac{1}{\sqrt{\pi}} \int_{-\infty}^{\infty} \frac{\exp \left(-x^{2}\right)}{x-\xi_{a}^{ \pm}} d t, \quad \Im\left(\xi_{a}^{ \pm}\right)>0,
$$

and $Z_{\kappa}\left(\xi^{ \pm}\right)$is the generalized modified dispersion function (Lazar, Schlickeiser \& Shukla 2008)

$$
\begin{aligned}
Z_{\kappa}\left(\xi_{e}^{ \pm}\right)= & \frac{1}{\pi^{1 / 2} \kappa_{e}^{1 / 2}} \frac{\Gamma\left(\kappa_{e}\right)}{\Gamma\left(\kappa_{e}-1 / 2\right)} \\
& \times \int_{-\infty}^{\infty} \frac{\left(1+x^{2} / \kappa_{e}\right)^{-\kappa_{e}}}{x-\xi_{e}^{ \pm}} d x, \quad \Im\left(\xi_{e}^{ \pm}\right)>0 .
\end{aligned}
$$

In the next section, we analyse the heat flux unstable solutions of the dispersion equation (7). RH solutions covert into LH modes when $\omega_{r}$ becomes negative, and same convention applies to $\mathrm{LH}$ solutions. The basic set of plasma parameters used in our numerical computations is tabulated in Table 1, unless otherwise noted. If we assume that suprathermal beam population incorporates both the halo and strahl components, the relative number density $\delta$ does not vary much with helioscentric distance (see figs 4 and 8 in Štverák et al. 2009), and here we adopt an average value $\delta=0.05$. For the other key parameters we consider values typically encountered in the solar wind at 1 au, e.g. $\beta_{c} \simeq \beta_{p} \geqslant 0.1$ (Štverák et al. 2008). Lower values (e.g. $\beta_{c} \simeq \beta_{p}=0.04$ ) may also be assumed for comparison with previous studies. High values of $\kappa$-index, e.g. $\kappa \geqslant$ 4 , are more specific to low heliospheric distances $(\leqslant 1 \mathrm{au})$, while low values, e.g. $\kappa<4$, are generated with the expansion of the solar wind beyond 1 au (Štverák et al. 2009; Pierrard et al. 2016). Here we show that suprathermal effects may be noticeable even for a high $\kappa \geqslant 6$, usually assimilated to a Maxwellian representation (Saeed et al. 2017b).

\section{INSTABILITY OF WHISTLER MODES}

In this section, we study the WHF instability driven by the corebeam counterstreaming electrons described above, for a wide range of parameters typically encountered in space plasmas. The unstable solutions are derived numerically starting from the dispersion relation (7) for $\mathrm{RH}$ modes (for $\xi_{p}^{+}$). We consider two alternative representations for the beam component. First, we assume both counter-beaming populations Maxwellian distributed, which is a common approach in the literature, see Gary (1985), Saeed et al. (2017a), and references therein. In this case the beam is a drifting Maxwellian recovered from a drifting Kappa in equation (4), in the limit $\kappa \rightarrow \infty$. In the second part, we will consider a scenario more realistic for the solar wind conditions, when the electron beam is reproduced by a drifting Kappa and instability conditions may be altered by the suprathermal electrons (Lazar et al. 2016). (a) $\delta=0.05, \beta_{\mathrm{c}}=0.1, \beta_{\mathrm{p}}=\beta_{\mathrm{c}}$

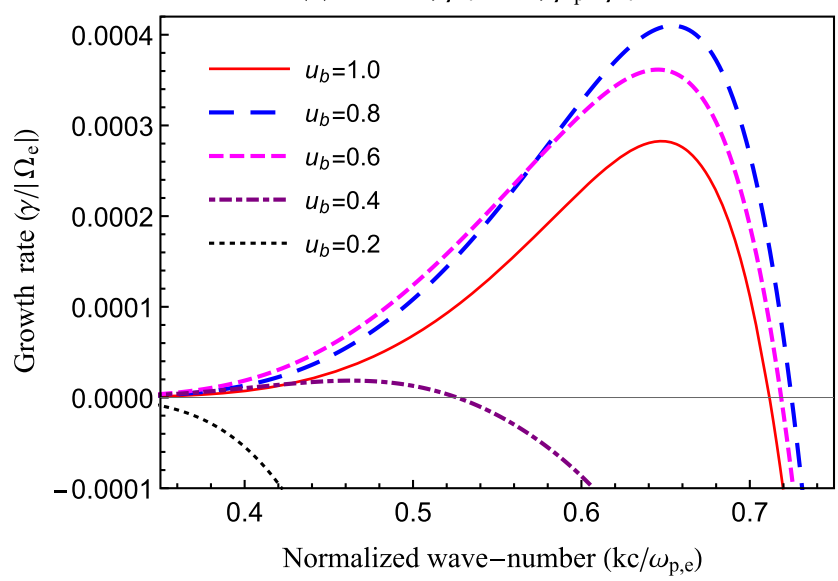

(b) $\delta=0.05, \beta_{\mathrm{c}}=0.1, \beta_{\mathrm{p}}=\beta_{\mathrm{c}}$

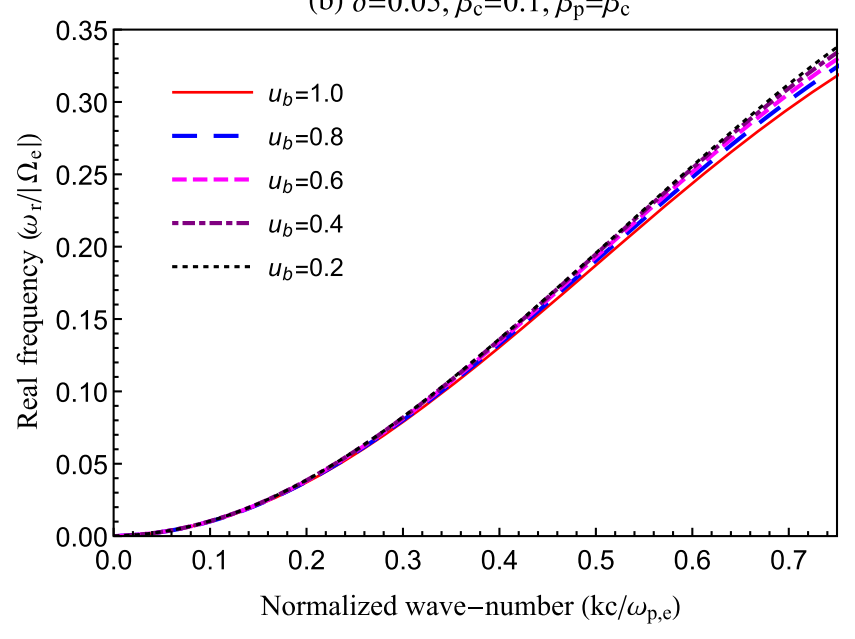

Figure 1. Effect of the beam velocity $u_{b}=[0.2-1.0]$ on the WHF instability growth rate (panel a) and wave frequency (panel b).

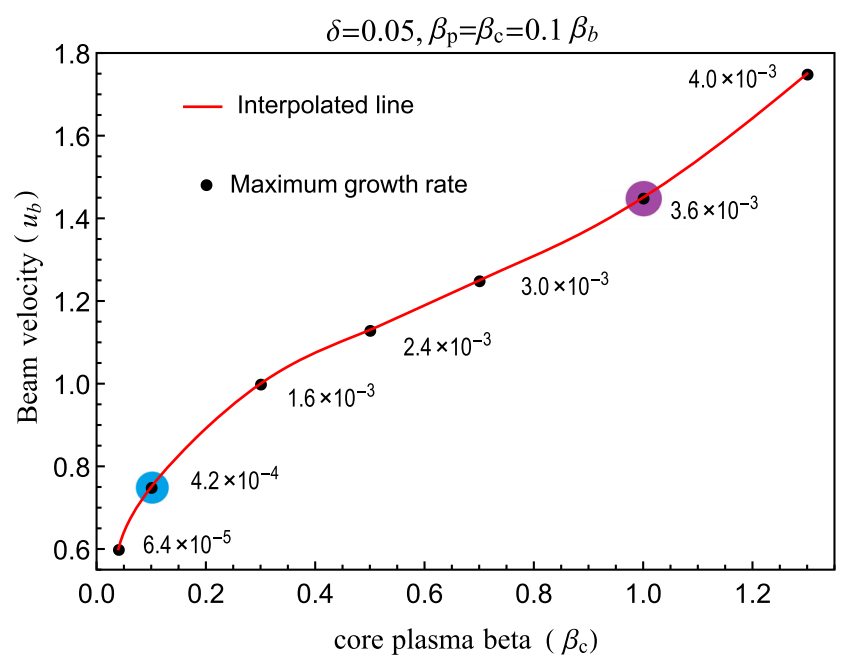

Figure 2. Maximum growth-rate conditions in terms of the beam speed and the core plasma beta. 
(a) $\delta=0.05, U_{b} / \mathrm{c}=0.0075, \beta_{\mathrm{c}}=0.1, \beta_{\mathrm{p}}=\beta_{\mathrm{c}}$

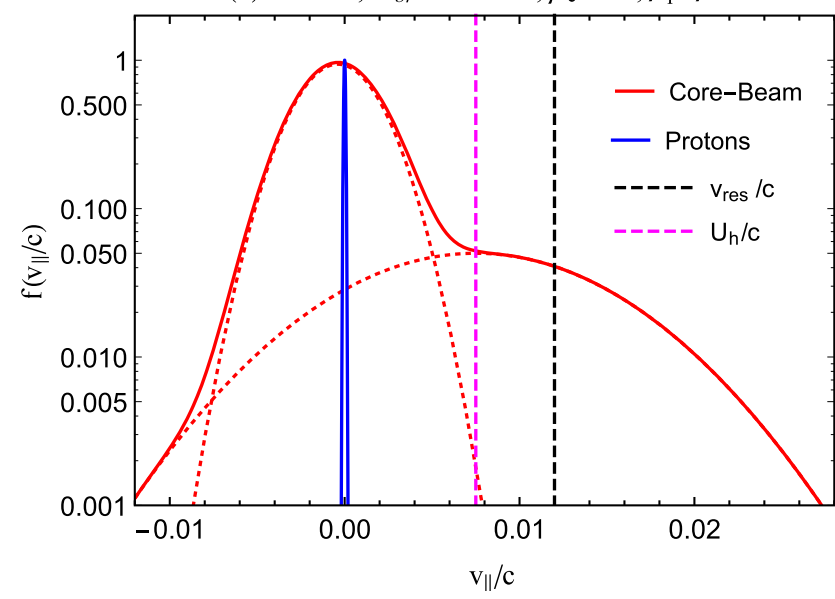

(b) $\delta=0.05, U_{b} / \mathrm{c}=0.02, \beta_{\mathrm{c}}=0.1, \beta_{\mathrm{p}}=\beta_{\mathrm{c}}$

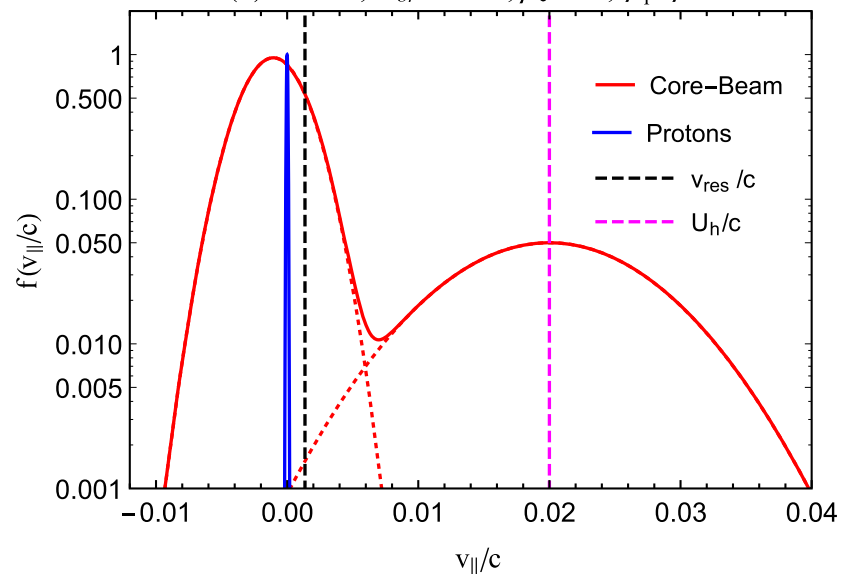

Figure 3. Velocity distributions susceptible to the WHF instability for $u_{b}$ $=0.75$ in panel (a), and $u_{b}=2.0$ in panel (b).

Table 1. Plasma parameters in the present study.

\begin{tabular}{lccc}
\hline & Beam electrons $(b)$ & Core electrons $(c)$ & Protons $(i)$ \\
\hline$n_{j} / n_{i}$ & 0.05 & 0.95 & 1.0 \\
$\beta_{j, 11} / \beta_{i, \|}$ & 10.0 & 1.0 & 1.0 \\
$m_{j} / m_{i}$ & $1 / 1836$ & $1 / 1836$ & 1.0 \\
$\kappa$ & $3, \infty$ & $\infty$ & $\infty$ \\
$\beta_{j, \perp} / \beta_{j, \|}$ & 1.0 & 1.0 & 1.0 \\
\hline
\end{tabular}

\subsection{Maxwellian beam}

Fig. 1 describes the influence of different beaming velocities $u_{b}$ $=1.0,0.8,0.6,0.4,0.2$ on the WHF growth rates (panel a) and wave frequency (panel b), assuming $\delta=0.05$ and $\beta_{c}=\beta_{p}=0.1$. Growth rates show a non-uniform variation, increasing and then decreasing with increasing $u_{b}$, and maximum growth rate (highest peak) is reached for $u_{b} \simeq 0.8$. The corresponding wave frequencies in Fig.1(b) are positive $\omega_{r}>0$, i.e. RH polarization, and slightly decrease by increasing the beaming velocity. In these examples, the instability is driven by the relative counterstreaming motion of electron components (Gary 1985). In order to identify the dominance regime of WHF instability, in Fig. 2 we show the beam velocity threshold $u_{b t}$ required for the instability to display maximum growth rates, as a function of the core plasma beta $\beta_{c}$. Physical conditions are provided for the fastest growing modes, explicitly indicating the maximum growth rates $\gamma_{m} /\left|\Omega_{e}\right|$ that increase with $\beta_{c}$ and $u_{b}$, as also the electrons become resonant. Moreover, Fig. 2 shows that the beam velocity threshold $u_{b t}$ increases as the core beta increases. The correlation between growth rates and the driver, e.g. beaming velocity $u_{b}$, is suggested by the resonance condition: whistlers are destabilized by the resonant electrons satisfying $\left|\xi_{b}^{+}\right| \simeq 1$, which implies $\left|\Omega_{e}\right|=k V_{t h \text {, res }}-k U_{b}>0$. Thus, the resonant instability requires $U_{b}<V_{t h}$, and Fig. 3 presents two counter-beaming electron distributions (red lines) satisfying this condition, and non-streaming Maxwellian protons (blue lines). Plasma parameters are same as in Fig. 1, except the normalized beaming velocity $u_{b}=U_{b} \omega_{e} /\left(c\left|\Omega_{e}\right|\right)$ $=0.75$ (required for the instability to display maximum growth rate) in panel (a) and $u_{b}=2.0$ in panel (b) $\left(\omega_{e} /\left|\Omega_{e}\right|=100\right)$. For the core-beam electrons we chose $\alpha_{\|} / c=\sqrt{\beta_{c}} \omega_{e} /\left|\Omega_{e}\right|=0.0032\left(\beta_{c}\right.$ $=0.1$ ) and $\theta_{\|} / c=\sqrt{\beta_{b}} \omega_{e} /\left|\Omega_{e}\right|=0.01$ (with $\beta_{b}=1.0$ ), while for the stationary protons $\alpha_{\|} / c=\sqrt{\beta_{p} / \mu} \omega_{e} /\left|\Omega_{e}\right|=7.3 \times 10^{-5}\left(\beta_{p}\right.$ $\left.=\beta_{c}=0.1\right)$. For a beaming velocity $u_{b}=0.75$ the resonant electrons have $v_{\text {res }}=V_{\text {res }} \omega_{e} /\left(c\left|\Omega_{e}\right|\right)=1.2$ (panel a), while for $u_{b}=2.0$ we find $v_{\text {res }}=0.136$ (panel $\mathrm{b}$ ). The resonant velocity $v_{\text {res }}$ in panel (a) is slightly higher than the beaming velocity $u_{b}$ involving more electrons from the beam population and enhancing the instability. The unstable solution in this case corresponds to the blue shaded point in Fig. 2 with maximum growth rate $\gamma_{m}=4.2 \times 10^{-4}\left|\Omega_{e}\right|$. By contrast, in panel (b) the resonant electrons with $v_{\text {res }}=0.136$ (much lower than $u_{b}=2.0$ ) are located in the core, and (maximum) growth rate is reduced to $\gamma_{m}=2.5 \times 10^{-6}\left|\Omega_{e}\right|$.

At this stage, an important question should be answered. The nonuniform variation of maximum growth rates (which increase and then decrease) with increasing beaming velocity $u_{b}$, prompts us to question the role played by this instability in the relaxation of more energetic beams with $u_{b}>u_{b t}$. Fig. 4 presents growth rates (panel a) and wave frequency (panel $b$ ) of HF instabilities for the same plasma parameters as in Fig. 2, for $\beta_{c}=1.0$ (purple shaded point), but for a higher beaming velocity $u_{b}=5$. In this case the growth rate displays two distinct peaks, one of the electron FHF instability at low wave numbers, and a second peak corresponding to WHF instability at larger wave numbers. The wave frequency in panel (b) confirms the LH polarization of the FHF modes (the zoom-in subplot), which converts to an RH polarization of the WHF peak. If the density contrast $\delta$ is constant, this transition between the WHF and FHF instabilities depends only on the plasma beta $\beta_{c}$ and the beaming velocity $u_{b}$. Similar transition has been reported by Gary et al. (1984) between the LH and RH modes driven unstable by the EM ion beam instabilities (RH non-resonant ion beam instability obtained for low beam-core temperature contrast $T_{b} / T_{c}=10$, converts to a resonant LH mode for higher $T_{b} / T_{c}=100$ (see figs 2 and 7 in Gary et al. 1984). The interplay between these growing modes will be discussed here next after identifying the FHF instability conditions in Section 4.

In order to understand the transition between the WHF and FHF instabilities, in Fig. 5 we display two distinct VDFs, in panel (a) the one used to derive the WHF solution corresponding to the purple shaded point in Fig. 2, and in panel (b) the VDF at the origin of two peaks in Fig. 4. In panel (a) the distribution is typical for WHF solutions with maximized growth rates, while panel (b) shows a distribution relevant for the transition between WHF and FHF, as already described in Fig. 4. These contours are plotted for different beaming velocities $u_{b}=1.45$ in panel (a) and $u_{b}=5.0$ in panel (b), but for the same plasma beta parameters, i.e. $\beta_{c}=1.0$ and $\beta_{b}$ $=10.0$. For a small $u_{b}=1.45$ the contrast between core and beam is modest, see the contour level 0.045 (orange colour) in panel (a), and these components can be considered 'strongly coupled', as a singular population. For a higher $u_{b}=5.0$ in panel (b), the beam 
(a) $\delta=0.05, \beta_{\mathrm{c}}=1.0, \beta_{\mathrm{p}}=\beta_{\mathrm{c}}$

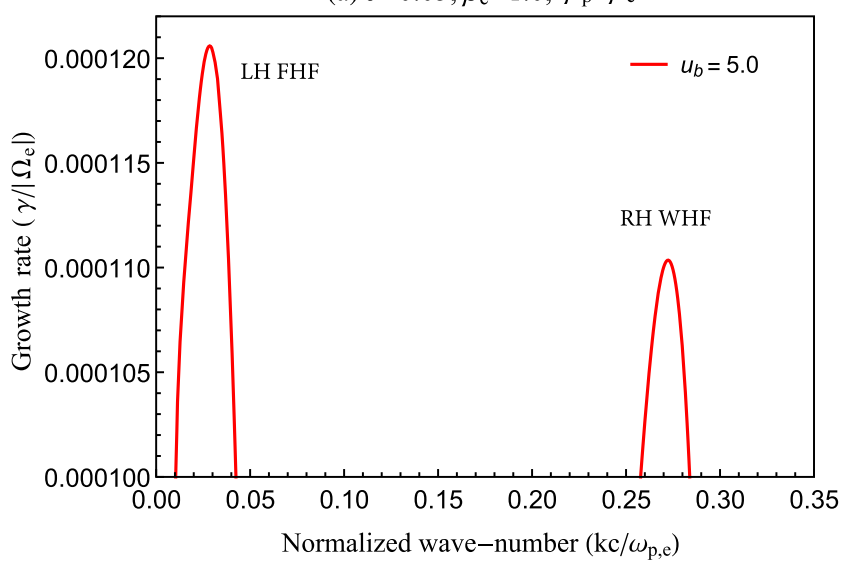

(b) $\delta=0.05, \beta_{\mathrm{c}}=1.0, \beta_{\mathrm{p}}=\beta_{\mathrm{c}}$

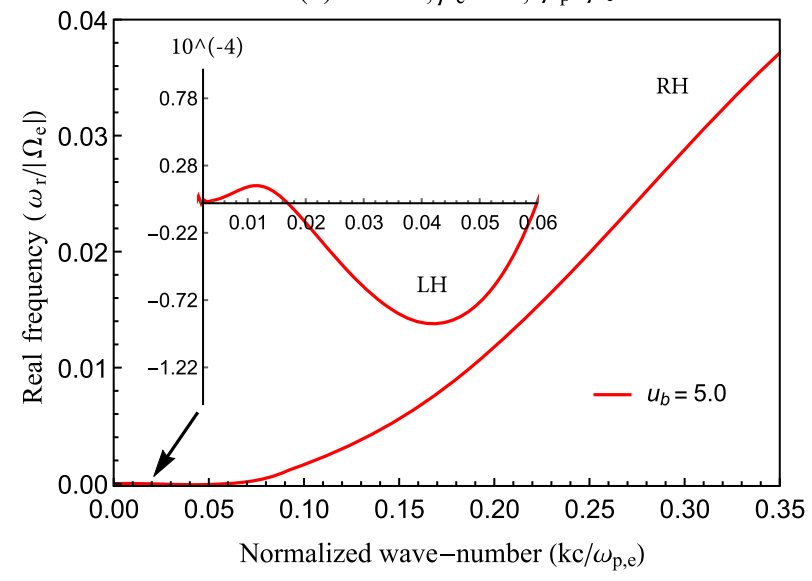

Figure 4. Transitive regime with both peaks of RH WHF and LH FHF growth rates in panel (a), and different polarizations indicated by the wave frequency in panel (b).

displays a peak markedly departed from the core peak, see the contour level 0.045 (orange colour). In this case, the beam appears 'weakly coupled' and any further increase of beaming velocity in parallel direction implies an effective increase of the anisotropy in this direction, which must be favorable to an LH FHF instability. Panels (a) in Figs 3 and 5 show the distributions corresponding to the blue $\left(\beta_{c}=0.1\right)$ and purple $\left(\beta_{c}=1.0\right)$ shaded points in Fig. 2. A comparison becomes straightforward and should explain the increase of the beaming velocity threshold $u_{b t}$ with $\beta_{c}$. In Fig. 5 (a) the electrons have thermal velocities $v_{\|} / c$ much higher than those in Fig. 3 (a) (assuming the plasma beta increasing with thermal velocity), and WHF instability is excited for higher beaming velocities, i.e. $u_{b}=1.45$.

\subsection{Kappa distributed beam}

In a more realistic solar wind scenario the suprathermal beam population is better reproduced by a drifting Kappa, as given in equation (4). Fig. 6(a) describes the effect of suprathermal electrons on the WHF solutions by varying the power index $\kappa=3,4,6,8, \infty$. The other parameters (kept constant in the analysis) are $u_{b}=0.6$, $\beta_{c}=0.04, \beta_{p}=\beta_{c}=\beta_{b} / 10$, and density contrast $\delta=0.05$. The suprathermal electrons in the beam have a stimulating effect on the WHF, enhancing growth rates and increasing the range of unstable wave numbers. These results are in agreement with kinetic simu-
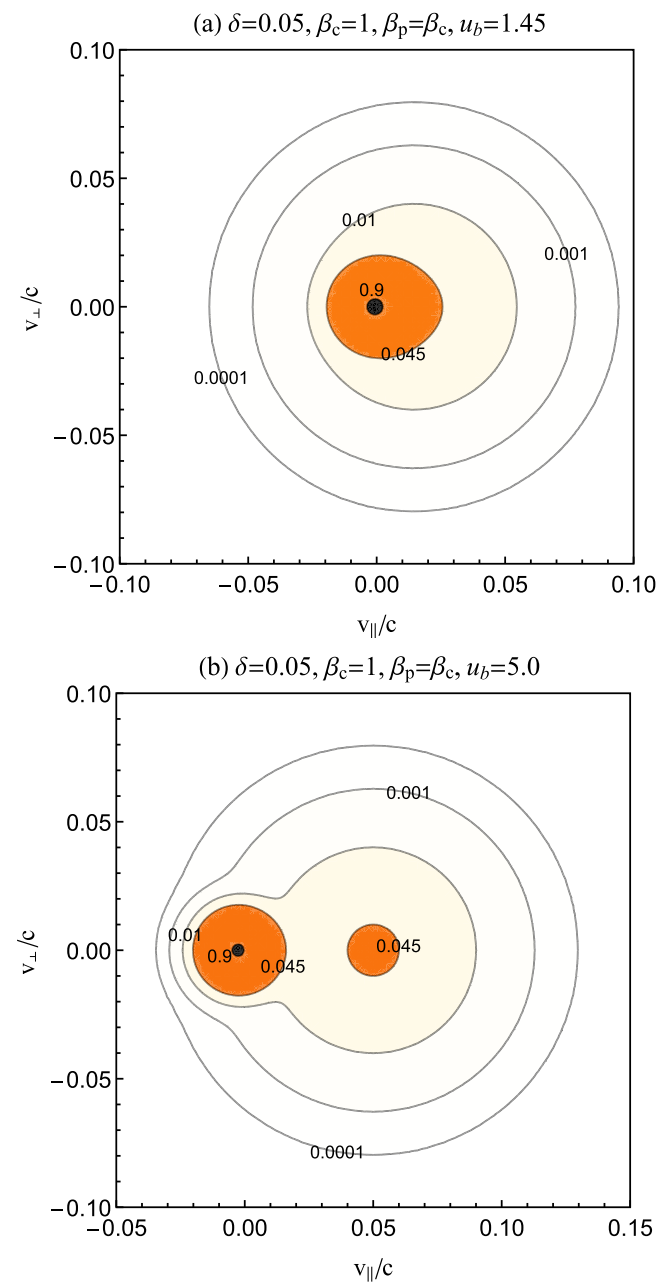

Figure 5. Beam-plasma distributions susceptible to the WHF instability $\left(u_{b}=1.5\right)$ in panel (a), and the FHF instability $\left(u_{b}=5.0\right)$ in panel (b).

lations, which suggest that the heat flux in the outer corona and solar wind is stimulated by the electron suprathermal populations (Landi \& Pantellini 2001). The wave frequency (not shown here) remains roughly unchanged to the variation of $\kappa$. Panel (b) provides a more general picture on the maximum growth rates varying with the beaming velocity threshold $u_{b t}$ and the power index $\kappa=3,6$, $\infty$ (for $\beta_{c}=0.04$ and $\delta=0.05$ ). The maximum growth rates are markedly enhanced with the abundance of suprathermal population in the beam, i.e. with decreasing $\kappa$. For $\kappa=3$ the maximum growth rate exhibits a peak five times greater than that obtained for Maxwellian $(\kappa \rightarrow \infty)$ counterstreams. As a consequence of that, conditions favorable to WHF instabilities extend to markedly higher beaming velocities, roughly four times higher than that needed by Maxwellian electron beams to stabilize. Beaming velocity thresholds $u_{b t}$, associated with different maximum growth rates, increase in the presence of suprathermal beaming electrons, i.e. decreasing $\kappa$ (see the cyan shaded area).

\section{BEAMING FIREHOSE INSTABILITY}

In this section, we investigate the second branch of HF instabilities, namely, the beaming firehose, also known as the FHF instability, which develops for higher beaming velocities $u_{b}$. The unstable modes are LH polarized and can be obtained from a conversion of 
(a) $\delta=0.05, \beta_{\mathrm{c}}=0.04, \beta_{\mathrm{p}}=\beta_{\mathrm{c}}, u_{b}=0.6$

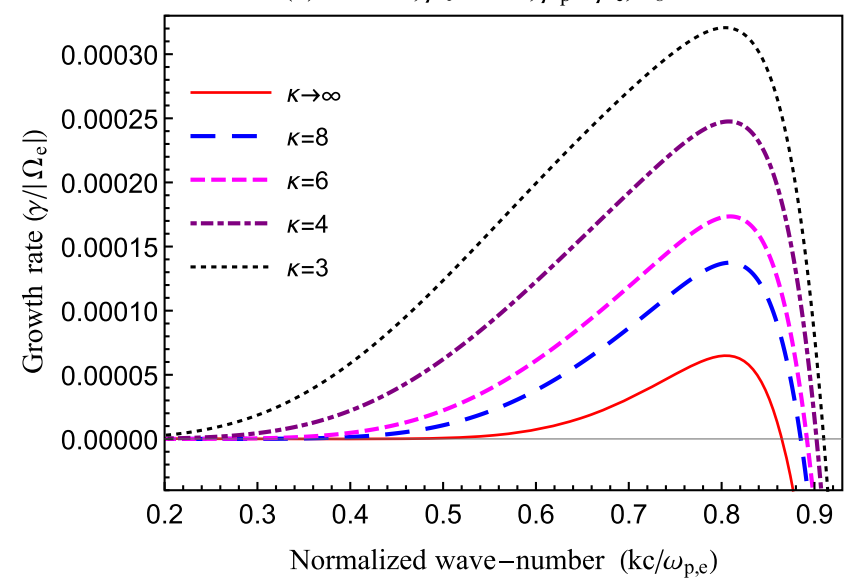

(b) $\delta=0.05, \beta_{\mathrm{c}}=1.2, \beta_{\mathrm{p}}=\beta_{\mathrm{c}}$

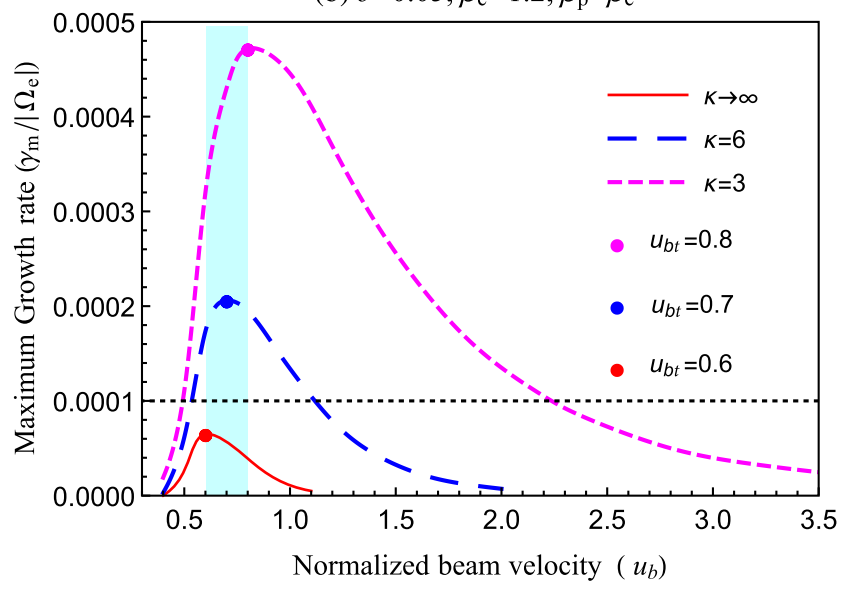

Figure 6. Effect of the power index $\kappa$ on the WHF instability: growth rate in panel (a), and maximum growth rates (as a function of the beam velocity $u_{b}$ ) in panel (b).

RH modes with increasing $u_{b}$ (see the analysis in Fig. 4), or starting directly from the dispersion relation (7) for LH modes $\left(\xi_{p}^{-}\right)$.

\subsection{Maxwellian beam}

We start again with an analysis of an idealized situation when both counter-beams of electrons are Maxwellian. The dispersion relation (7) for LH modes is solved numerically in the limit of $\kappa \rightarrow \infty$. From the variation with beaming velocity we can identify the instability conditions and mode polarization (given by the sign of $\omega_{r}$ ). Recent studies of FHF instability have restricted to a low $\beta$ regime i.e. $\beta_{c}=0.04$ (Saeed et al. 2017a), although firehose instability is significantly more efficient for high $\beta_{c}>1$ regimes. In Fig. 7 we present FHF solutions for a relatively high core plasma beta $\beta_{c}=$ 1.2. Panel (a) shows growth rates increasing as the beaming velocity $u_{b}$ increases, while the range of unstable wave numbers increases towards lower values. By comparison to whistlers these FHF modes are destabilized at lower wavenumbers (i.e. higher proton scales). The corresponding wave frequencies in panel (b) are markedly increased by increasing $u_{b}$, and remain positive $\omega_{r}>0$ in the range of FHF peaks. The conversion from RH modes is still visible for the less-energetic beams, i.e. for $u_{b}=3.3$. (a) $\delta=0.05, \beta_{\mathrm{c}}=1.2, \beta_{\mathrm{p}}=\beta_{\mathrm{c}}$

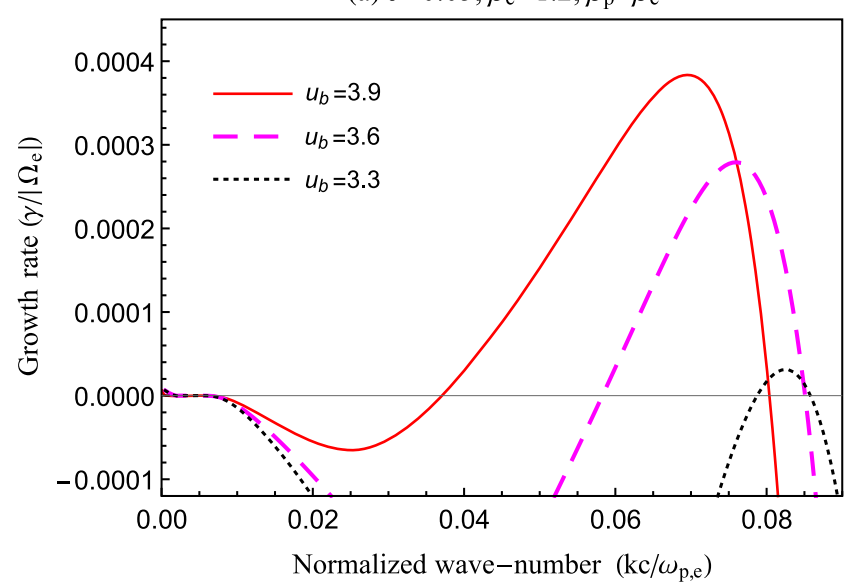

(b) $\delta=0.05, \beta_{\mathrm{c}}=1.2, \beta_{\mathrm{p}}=\beta_{\mathrm{c}}$

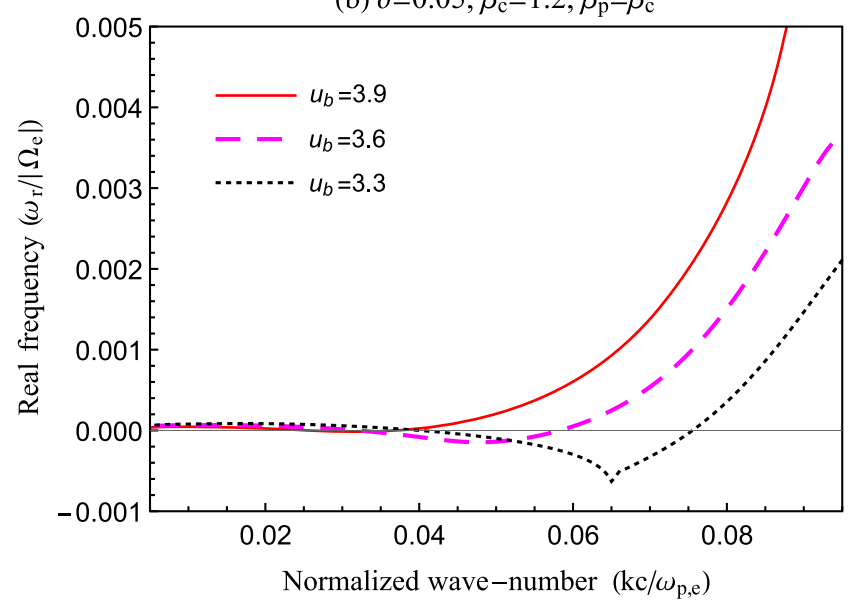

Figure 7. Effect of the beaming velocity $u_{b}$ on the FHF instability: growth rates in panel (a) and wave frequency in panel (b).

\subsection{Kappa distributed beam}

In the presence of suprathermals the electron beam is well described by a drifting-Kappa distribution function. Fig. 8 displays growth rates (panel a) and wave frequency (panel b) of FHF instability and their variation with $\kappa$. Plasma parameters are same as in Fig. 7, with $u_{b}=3.6$. Panel (a) clearly shows that suprathermal electrons in the beam have an inhibiting effect on the FHF instability, i.e. the growth rates and the range of unstable wave numbers decrease with decreasing the power index $\kappa$. The corresponding wave frequencies in panel (b) are enhanced in the presence of suprathermal electrons, i.e. decreasing $\kappa$. These effects have not been reported by Saeed et al. (2017a), who restricted to low $\beta$ regimes, and to Kappa approaches with $\kappa$-independent temperatures. Here we find that FHF instability is inhibited by the suprathermal electrons, by contrast to whistlers that are stimulated by the same suprathermals. In Fig. 9 we show explicitly the VDFs invoked to derive the unstable solutions in Fig. 8, for a Maxwellian beam $(\kappa \rightarrow \infty)$ in panel (a), and a Kappa beam $(\kappa=3)$ in panel (b) $\left(\beta_{c}=\beta_{p}=1.2\right.$ and $\left.\beta_{b}=12\right)$. The beam-core contrast is diminished in the presence of suprathermals (compare the orange contour in panels (a) and (b), which may explain the inhibition of FHF instability and the stimulation of whistlers, as also shown by the velocity thresholds derived in the next section. 


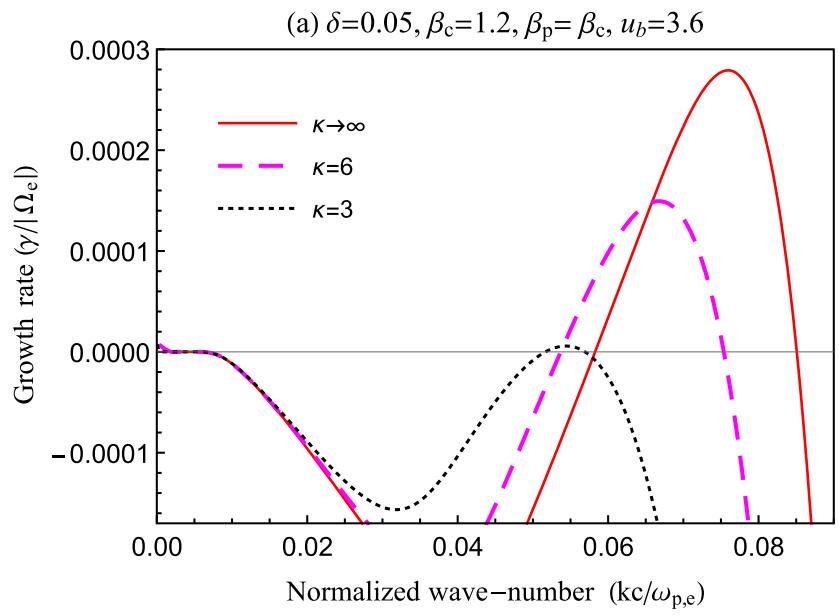

(b) $\delta=0.05, \beta_{\mathrm{c}}=1.2, \beta_{\mathrm{p}}=\beta_{\mathrm{c}}, u_{b}=3.6$

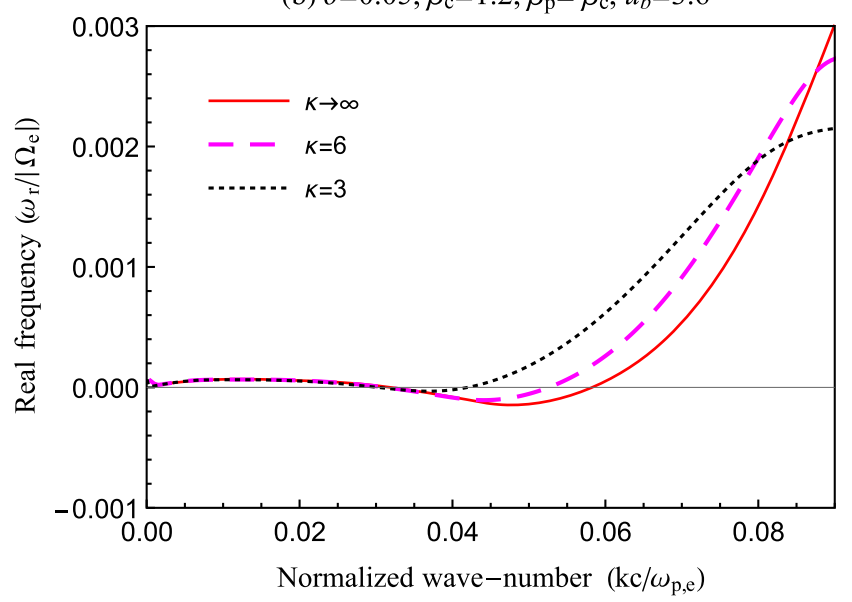

Figure 8. Effect of the power index $\kappa$ on the FHF instability: growth rate in panel (a), and wave frequency in panel (b).

\section{INSTABILITY THRESHOLDS}

Figs $10-12$ present the beam velocity thresholds, which allow us to identify the regime of dominance of each instability, and the transitive conditions triggering the mode conversion. We know already that WHF instability is limited to low beam velocities (non-uniform variation of growth rates with $u_{b}$ ), and it is therefore expected to develop between the upper and lower thresholds which are displayed in Fig. 10, panels (a) and (b), respectively. These thresholds are derived for a maximum growth rate $\gamma_{m}=10^{-4}\left|\Omega_{e}\right|$ approaching the marginal stability $\left(\gamma_{m}=0\right)$, in terms of the main plasma parameters conditioning the instability, the beam velocity $\left(u_{b}\right)$, and the core plasma beta $\left(\beta_{c}\right)$. Contours are obtained with an inverse power law

$u_{b}=\frac{a}{\beta_{c}^{b}}$,

where $a$ and $b$ are the fitting parameters tabulated in Table 2. The most unstable regimes for the WHF modes are indicated by the dashed arrows, and are found between the upper and lower thresholds.

The effects of suprathermal beaming electrons are shown by contrasting thresholds obtained for a Maxwellian beam (red contours) with those for a Kappa distributed beam with $\kappa=3$ (blue contours). In panel (a) the increase of the upper threshold with the core plasma beta means an extent of the conditions favorable to WHF instability to higher values of beam velocity. Moreover, the upper threshold (a) $\delta=0.05, \beta_{\mathrm{c}}=1.2, \beta_{\mathrm{p}}=\beta_{\mathrm{c}}, u_{b}=3.6$

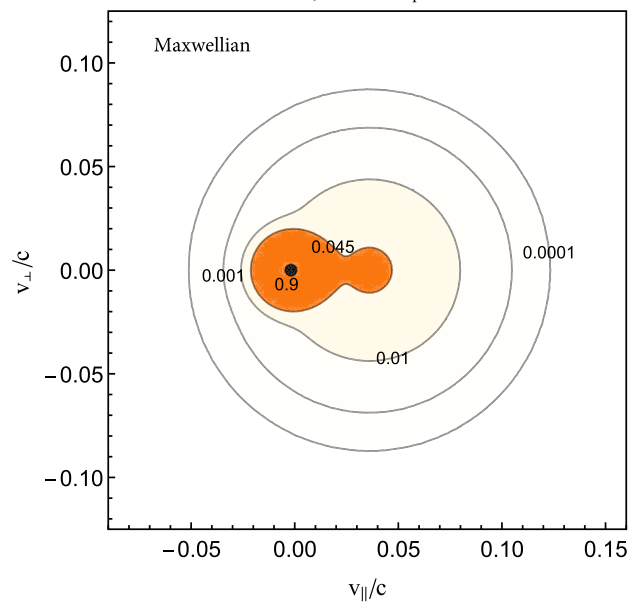

(b) $\delta=0.05, \beta_{\mathrm{c}}=1.2, \beta_{\mathrm{p}}=\beta_{\mathrm{c}}, u_{b}=3.6$

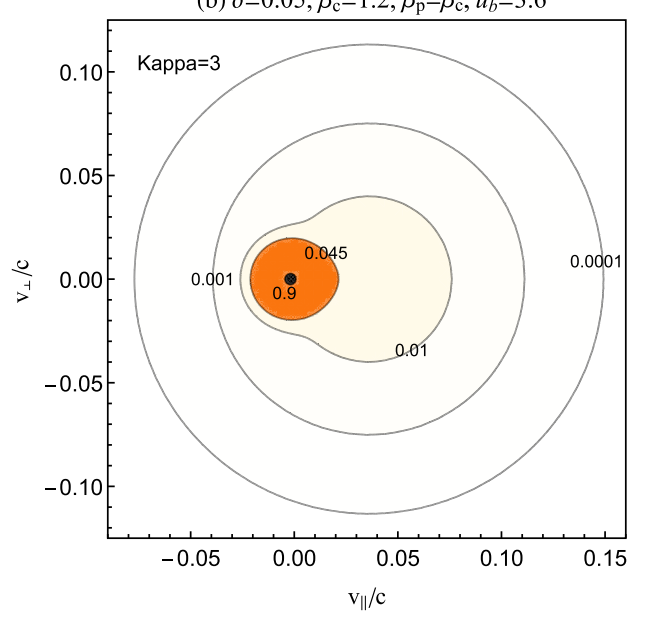

Figure 9. Velocity distributions susceptible to FHF instability in Fig. 8 with a Maxwellian $(\kappa \rightarrow \infty)$ beam in panel (a), and a Kappa $(\kappa=3)$ beam in panel (b).

is markedly enhanced in the presence of suprathermal electrons, i.e. for $\kappa=3$, further extending the instability conditions to even more energetic beams. Contrary to the upper thresholds, the lower thresholds in panel (b) decrease as the core beta increases, confirming the results in Fig. 2. Again, the WHF instability is stimulated by the suprathermal electrons (for $\kappa=3$, confirming the results in Fig. 6), in this case by decreasing the beam velocity thresholds but increasing susceptibility to this instability.

Fig. 11 presents the beam velocity thresholds derived for the FHF instability $\left(\gamma_{m}=10^{-4}\left|\Omega_{e}\right|\right)$ in terms of the core plasma beta $\beta_{c}$, and for the same plasma parameters as in Fig. 10. Contours are obtained by fitting the same law in equation (10) (see also Table 2). As explained before, the results in Fig. 5 suggest that for a higher thermal velocity (implying a higher $\beta_{c}$ ) we need more energetic beams, i.e. a higher $u_{b}$, to excite the FHF instability. Variation with the core plasma beta resembles that of the upper threshold of WHF instability, but the most unstable FHF regimes are located above the FHF thresholds, as indicated by the dashed arrows in Fig. 11. Moreover, these thresholds increase as the power index $\kappa$ decreases, confirming the inhibiting effect of the suprathermal beaming electrons on the FHF instability, described already in Fig. 8. 
(a) $\gamma_{\mathrm{m}} /\left|\Omega_{\mathrm{e}}\right|=10^{-4}, \delta=0.05$

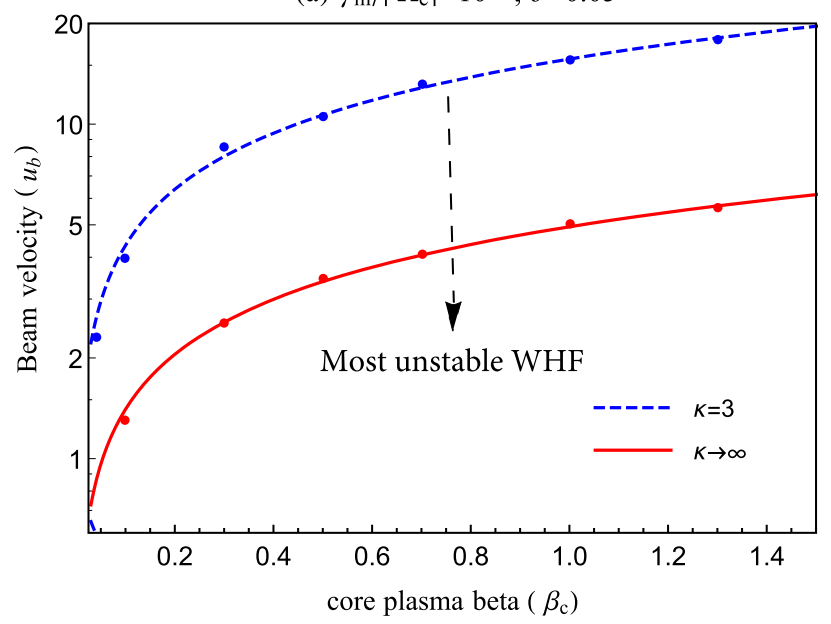

(b) $\gamma_{\mathrm{m}} /\left|\Omega_{\mathrm{e}}\right|=10^{-4}, \delta=0.05$

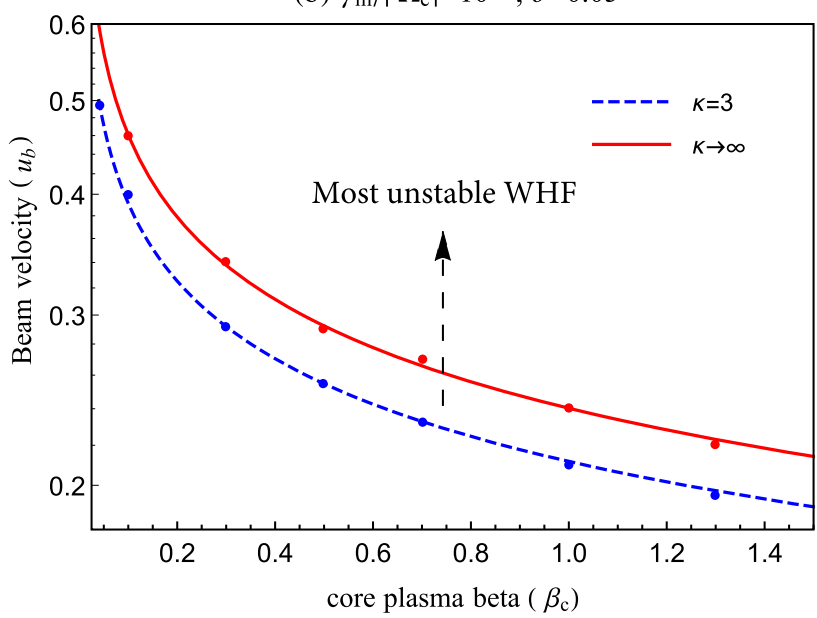

Figure 10. The beam velocity upper (a) and lower (b) thresholds for the WHF instability (maximum growth rate $\gamma_{m} /\left|\Omega_{e}\right|=10^{-4}$ ) driven by Maxwellian (red) and Kappa (blue) distributed beams.

Table 2. Fitting parameters in Figs 10 and 11.

\begin{tabular}{lcccccc}
\hline & Whistler (a) & \multicolumn{3}{c}{ Whistler (b) } & \multicolumn{3}{c}{ Electron firehose } \\
& $a$ & $b$ & $a$ & $b$ & $a$ & $b$ \\
\hline$\infty$ & 4.94 & -0.545 & 0.240 & 0.283 & 3.73 & -0.062 \\
3 & 15.6 & -0.557 & 0.212 & 0.266 & 3.34 & -0.073 \\
\hline
\end{tabular}

Now a comparison of the instability thresholds becomes straightforward, and enables to build a clear picture on the interplay of these instabilities and their regimes of dominance. As illustrated in Fig. 11 the most unstable FHF modes are obtained for beams with relatively high velocities $u_{b}>2.5$, making relevant only a contrast with the upper threshold of WHF instability. This contrast is provided in Fig. 12 for a Maxwellian beam $(\kappa \rightarrow \infty)$ in panel (a) and for a Kappa beam $(\kappa=3)$ in panel (b). Dashed arrows in Fig. 12 indicate the most unstable regime for each instability. Panel (a) shows clearly that the WHF instability is dominant at low beaming velocities, e.g. $u_{b}<2.5$, while for higher beam velocities the FHF instability arises and eventually dominates, if the core plasma beta is low enough, e.g. $\beta_{c}<0.5$, or if the beam is energetic enough, with high $u_{b}$ exceeding the threshold of WHF. If $\beta_{c}>0.5$ is large enough

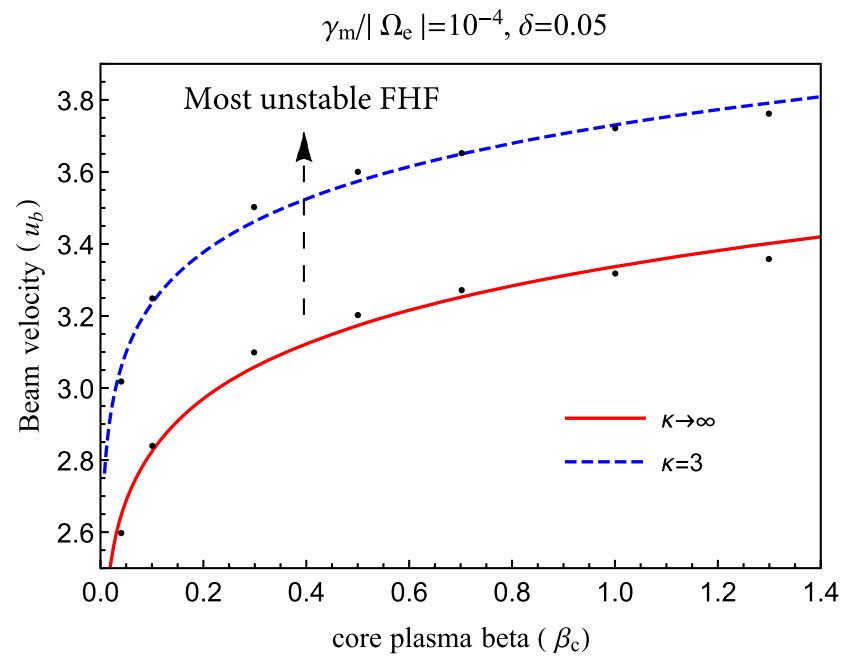

Figure 11. The beam velocity threshold for the FHF instability $\left(\gamma_{m} /\left|\Omega_{e}\right|\right.$ $=10^{-4}$ ) driven by Maxwellian (red) and Kappa (blue) distributed beams.

(a) $\gamma_{\mathrm{m}} /\left|\Omega_{\mathrm{e}}\right|=10^{-4}, \delta=0.05, \kappa \rightarrow \infty$

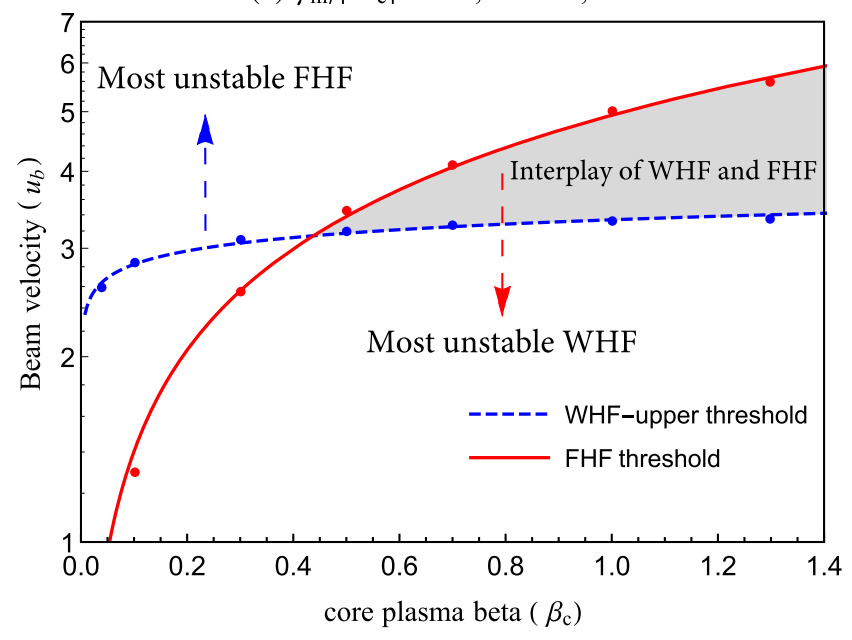

(b) $\gamma_{\mathrm{m}} /\left|\Omega_{\mathrm{e}}\right|=10^{-4}, \delta=0.05, \kappa=3$

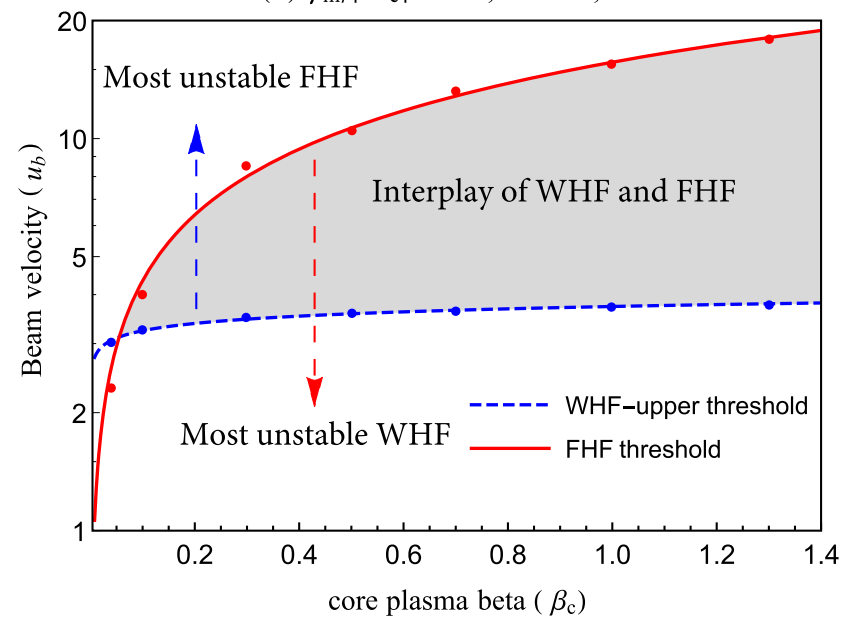

Figure 12. Comparison of the instability thresholds $\left(\gamma_{m} /\left|\Omega_{e}\right|=10^{-4}\right)$ : WHF (red) vs. FHF (blue) for (a) Maxwellian and (b) Kappa beams. 
we identify a regime where both WHF and FHF instabilities may coexist, i.e. grey area between their thresholds. We have already shown a representative case for the interplay of these two instabilities in Fig. 4. The interval of beam velocities relevant for this regime of transition and interplay increases with the core plasma beta. This regime further expands in the presence of the suprathermal beaming electrons, see panel (b) $(\kappa=3)$, when the limit value of the core plasma beta becomes also significantly lower, $\beta_{c}>0.05$.

\section{CONCLUSIVE DISCUSSIONS}

In this paper, we have clarified the main contrasting properties of heat flux instabilities, namely the WHF and FHF instabilities, driven by the electron beam-core relative drift. Thermal and suprathermal spread of electrons plays a key role, and here we have assumed isotropic temperatures, focusing on the effect of beaming electrons as a unique source of free energy. In an attempt to overcome the limitations from previous studies and reproduce conditions typically encountered in space plasmas, here we have considered an extended range of values for the key plasma parameters, e.g. the beam velocity and the core plasma beta, and for the electron beam a more realistic (drifting-)Kappa distribution enabling a direct contrast to the Maxwellian limit of lower temperature.

Less-energetic beams destabilize the whistler modes in the wavefrequency range $\Omega_{p} \ll \omega_{r} \ll\left|\Omega_{e}\right|$. This is the WHF instability with growth rates conditioned by the thermal velocity of the resonant beam (Figs 2 and 3), and non-uniformly varying with the beam velocity (Fig. 1). The fastest growing modes are described by the maximum growth rates derived in Fig. 2 in terms of beam velocity threshold $u_{b t}$ and the core plasma beta parameter $\beta_{c}$. Growth rates are maximized by increasing any of these two parameters. Beaming velocities higher than this threshold can excite the LH-FHF growthrates, which display an additional peak at lower wave numbers (see Fig. 4). A qualitative explanation for this transitive regime is provided by the contours of the distributions in Figs 3 and 5, which show that beaming electrons become less resonant for higher beaming velocities, inhibiting the WHF modes and exciting the FHF instability. These results confirm the earlier predictions in Gary (1985). The FHF instability has an opposite LH polarization and is excited for higher beaming velocities. This unstable mode may evolve out of an RH whistler mode for significant beaming velocities and/or core plasma beta parameters. Further increase of the beam velocity leads to a uniform increase of growth rates and wave frequencies. These transitions and conversions of the wave polarization are presented in Figs 7 and 8.

Electron heat flux is transported away from the solar corona mainly by the suprathermal strahl (or beaming) component with a drifting-Kappa distribution. According to our knowledge, in the existing studies of heat flux instabilities the effects of suprathermals are underestimated assuming Maxwellian beams or Kappa beams of comparable (kinetic) temperature, e.g. in Saeed et al. (2017a). However, in a realistic Kappa approach as the one invoked here, suprathermal electrons contribute with an excess of kinetic (free) energy (Lazar et al. 2015, 2017a) that stimulates WHF instability by enhancing the growth rates and extending the instability regime to higher beaming velocities, e.g. in Fig. 6. For more energetic beams susceptible to FHF instability, the same suprathermal electrons have an opposite effect, diminishing the growth rates and the range of unstable wave numbers. Physical explanations are suggested by the contours of the distributions in Fig. 9, which show that the beam-core contrast, and, implicitly, the effective anisotropy in the parallel direction are reduced in the presence of suprathermal electrons, causing inhibition of FHF modes and stimulation of WHF instability.

These physical insights have enabled us to identify the regimes of dominance for each of these two instabilities, in terms of the instability thresholds derived in Figs 10-11. The most unstable WHF modes are located between two thresholds, namely, a lower and an upper threshold in Fig. 10, while the most unstable FHF modes are located above the threshold in Fig. 11. As a consequence of that, by increasing the core plasma beta, conditions favorable to WHF instability are enhanced while those favorable to FHF are reduced. These effects are markedly stimulated in the presence of the suprathermal beaming electrons. For a low beam velocity $u_{b} \leqslant 2.5$ the WHF instability is dominant and the FHF modes are damped, which totally agree with the results for FHF instability in Saeed et al. (2017b). However, for more energetic beams, we have identified the regime of transition and mode conversion, where both instabilities can develop and compete in the relaxation process. Marginally bounded by the FHF threshold and the upper WHF threshold, the range of beaming velocities associated with this regime is considerably enhanced with increasing $\beta_{c}$, and due to the abundance of suprathermal electrons (i.e. lowering $\kappa$ ).

To conclude, in this paper we have decoded the interplay of HF instabilities conditioned by the relative beaming velocity of two countermoving electron populations, and in particular we have unveiled new unstable regimes induced or/and stimulated by the suprathermal beaming electrons. Suprathermal Kappa-distributed electrons are ubiquitous in the solar wind and their effects support earlier predictions (Gary 1985; Gary \& Li 2000), which indicate the WHF instability as the most probable mechanism of regularization of the electron strahl. However, an extended analysis to include oblique modes, such as the aperiodic branch of firehose instability, may also be opportune, especially for energetic electron beams. Our present results do offer valuable tools for future investigations seeking realistic approach of the electron beams and their implications in specific conditions, e.g. fast winds and interplanetary shocks. These conditions should also include the effect of temperature anisotropy of electrons, which is often reported by the solar wind observations (Štverák et al. 2008) and, eventually, the reaction of protons to the low-frequency fluctuations developed by the firehose-like instabilities (Sarfraz et al. 2017; Yoon et al. 2017).

\section{ACKNOWLEDGEMENTS}

These results were obtained in the framework of the projects SCHL 201/35-1 (DFG-German Research Foundation), GOA/2015-014 (KU Leuven), G0A2316N (FWO-Vlaanderen), and C 90347 (ESA Prodex 9). S.M. Shaaban acknowledges support by an FWO Postdoctoral Fellowship (Grant No. 12Z6218N), and an FWO Travel grant for long stay aboard (Grant No. V419818N). The authors acknowledge useful discussions during the first meeting of the ISSI team "Kappa Distributions".

\section{REFERENCES}

Anderson B. R., Skoug R. M., Steinberg J. T., McComas D. J., 2012, J Geophys. Res. (Space Phys.), 117, A04107

Bale S., Pulupa M., Salem C., Chen C., Quataert E., 2013, ApJ, 769, L22

Feldman W. C., Asbridge J. R., Bame S. J., Montgomery M. D., Gary S. P., 1975, J. Geophys. Res., 80, 4181

Feldman W., Asbridge J., Bame S., Gosling J., Lemons D., 1978, J. Geophys. Res.( Space Phys.), 83, 5285 
Fried B., Conte S., 1961, The Plasma Dispersion Function, Academic Press, New York

Gary S. P., 1985, J. Geophys. Res., 90, 10815

Gary S. P., 1993, Theory of Space Plasma Microinstabilities. Cambridge Univ. Press

Gary S. P., Li H., 2000, ApJ, 529, 1131

Gary S. P., Feldman W. C., Forslund D. W., Montgomery M. D., 1975, J. Geophys. Res., 80, 4197

Gary S. P., Smith C. W., Lee M. A., Goldstein M. L., Forslund D. W., 1984, Phys. Fluids, 27, 1852

Graham G. A. et al., 2017, J. Geophys. Res. (Space Phys.), 122, 3858

Gurgiolo C., Goldstein M. L., Viñas A. F., Fazakerley A. N., 2012, Annales Geophysicae, 30, 163

Hammond C. M., Feldman W. C., McComas D. J., Phillips J. L., Forsyth R. J., 1996, A\&A, 316, 350

Lacombe C., Alexandrova O., Matteini L., Santolík O., Cornilleau-Wehrlin N., Mangeney A., de Conchy Y., Maksimovic M., 2014, ApJ, 796, 5

Landi S., Pantellini F., 2001, A\&A, 372, 686

Lazar M., Schlickeiser R., Shukla P. K., 2008, Phys. Plasmas, 15, 042103

Lazar M., Poedts S., Fichtner H., 2015, A\&A, 582, A124

Lazar M., Fichtner H., Yoon P. H., 2016, A\&A, 589, A39

Lazar M., Shaaban S., Poedts S., Štverák Š., 2017a, MNRAS, 464, 564

Lazar M., Pierrard V., Shaaban S., Fichtner H., Poedts S., 2017b, A\&A, 602, A44

Lazar M., Shaaban S. M., Fichtner H., Poedts S., 2018, Phys. Plasmas, 25, 022902

Levinson A., Eichler D., 1992, ApJ, 387, 212

Lin R. P., 1998, Space Sci. Rev., 86, 61

Maksimovic M., Zouganelis I., Chaufray J.-Y., Issautier K., Scime E. E., Littleton J. E., Marsch E., McComas D. J., Salem C., Lin R. P., Elliott H., 2005, J. Geophys. Res. (Space Phys.), 110, A09104

Nieves-Chinchilla T., Viñas A. F., 2008, J. Geophys. Res. (Space Phys.), 113, A02105

Pagel C., Crooker N., Larson D., 2005, Geophys. Res. Lett., 32, L14105

Pagel C., Gary S. P., de Koning C. A., Skoug R. M., Steinberg J. T., 2007, J. Geophys. Res. (Space Phys.), 112, A04103
Pavan J., Viñas A. F., Yoon P. H., Ziebell L. F., Gaelzer R., 2013, ApJ, 769, L30

Pierrard V., Maksimovic M., Lemaire J., 2001, Ap\&SS, 277, 195

Pierrard V., Lazar M., Poedts S., Štverák Š., Maksimovic M., Trávníček P., 2016, Sol. Phys., 291, 2165

Pilipp W., Miggenrieder H., Montgomery M., Mühlhäuser K.-H., Rosenbauer H., Schwenn R., 1987, J. Geophys. Res. (Space Phys.), 92, 1075

Pistinner S., Eichler D., 1998, MNRAS, 301, 49

Saeed S., Sarfraz M., Yoon P. H., Lazar M., Qureshi M. N. S., 2017a, MNRAS, 465, 1672

Saeed S., Sarfraz M., Yoon P. H., Qureshi M. N. S., 2017b, MNRAS, 4936, stx049

Saito S., Gary S. P., 2007, J. Geophys. Res. (Space Phys.), 112, A06116

Sarfraz M., Yoon P., Saeed S., Abbas G., Shah H., 2017, Phys. Plasmas, 24, 012907

Scime E. E., Bame S. J., Feldman W. C., Gary S. P., Phillips J. L., Balogh A., 1994, J. Geophys. Res.(Space Phys.), 99, 23401

Shaaban S., Lazar M., Poedts S., Elhanbaly A., 2016, J. Geophys. Res. (Space Phys.), 121, 6031

Shaaban S., Lazar M., Astfalk P., Poedts S., 2018, J. Geophys. Res. (Space Phys.), 123, 1754

Spitzer L., Härm R., 1953, Phys. Rev., 89, 977

Vocks C., Salem C., Lin R. P., Mann G., 2005, ApJ, 627, 540

Walsh A. P., Arridge C. S., Masters A., Lewis G. R., Fazakerley A. N., Jones G. H., Owen C. J., Coates A. J., 2013, Geophys. Res. Lett., 40, 2495

Yoon P., López R., Seough J., Sarfraz M., 2017, Phys. Plasmas, 24, 112104

Štverák Š., Trávníček P., Maksimovic M., Marsch E., Fazakerley A. N., Scime E. E., 2008, J. Geophys. Res. (Space Phys.), 113, A03103

Štverák Š., Maksimovic M., Trávníček P. M., Marsch E., Fazakerley A. N., Scime E. E., 2009, J. Geophys. Res. (Space Phys.), 114, A05104

This paper has been typeset from a $\mathrm{T}_{\mathrm{E}} \mathrm{X} / \mathrm{L} \mathrm{T} \mathrm{T} \mathrm{X}$ file prepared by the author. 